\title{
On the Existence and Uniqueness of the Formal Solution to a Singular Partial Differential Equation
}

\author{
Pingli $\mathrm{Li}^{1,}$, , Sihui $\mathrm{Li}^{2, \mathrm{~b}}$ \\ ${ }^{1}$ School of Mathematics and Physics, Bohai University, Jinzhou, 121013, China \\ ${ }^{2}$ School of Mathematics and Physics, Bohai University, Jinzhou, 121013, China \\ aemail: lipingli1210@163.com, bemail: 1652967707@qq.com
}

Keywords: Formal Power Series Solution; Gevrey Order in a Monomial; Asymptotic Expansion in a Monomial

\begin{abstract}
According to the theory used in researching certain type of doubly singular differential equations, by computing directly, we find out that a singular partial differential equation in two complex variables has a unique formal power series solution which is in a special form and whose coefficients can be worked out explicitly. By estimating coefficients of the solution, we prove this formal solution is 1-Gevrey in a monomial. This gives a way to study Gevrey order in a monomial for formal solutions to certain classes of differential equations (or systems).
\end{abstract}

\section{Introduction}

In [1], arbitrary formal solutions of inhomogeneous partial differential equations are considered. Because of the obvious symmetry of the two variables in their problem they solved, their treatment, in some sense, prefer one variable over the other. They shows that the above formal solutions could be computed explicitly and that Gevrey order of them could be determined.

Asymptotic expansion in a monomial was introduced in [2] and the corresponding theory was used to study the formal solution of a class of doubly singular differential systems. After that, asymptotic expansion theory in a monomial is frequently used in studying formal power series solutions of certain class of differential equations (or systems) [3][4][5].

In this paper a formal solution to a singular linear partial differential equation is worked out explicitly. Gevrey order in a monomial of it is computed according to it's formula. This gives an idea to research certain kind of singular partial differential equations.

\section{Calculation of the formal solution}

Consider the following singular partial differential equation

$$
x \frac{\partial u}{\partial x}-(1+a x y) y \frac{\partial u}{\partial y}+\alpha u=(p x+q y) f(x y)
$$

Where $u$ is the unknown function which is a function of two complex variables $x$ and $y$. Nonzero constants $a, p, q$ and $\alpha$ are complex numbers, and $\alpha$ could not be an integer. $f$ is a function of one complex variable which is analytic in a small neighborhood of the origin in the complex plane. Furthermore it satisfies the condition:

$$
f(0)=0, f^{\prime}(0) \neq 0
$$

Suppose $f$ has the following power series expansion at the origin

$$
f(z)=\sum_{n=1}^{+\infty} b_{n} z^{n}
$$

Formally, write the unknown $u$ as below:

$$
u(x, y)=\sum_{m, n=0}^{+\infty} u_{m n} x^{m} y^{n}
$$

Then 
$x \frac{\partial u}{\partial x}=\sum_{m, n=0}^{+\infty} m u_{m n} x^{m} y^{n}, \quad y \frac{\partial u}{\partial y}=\sum_{m, n=0}^{+\infty} n u_{m n} x^{m} y^{n}$

Inserting the preceding formulas into (1), yields:

$\sum_{m, n=0}^{+\infty}(m-n+\alpha) u_{m n} x^{m} y^{n}-\sum_{m, n=0}^{+\infty} a n u_{m n} x^{m+1} y^{n+1}=\sum_{n=1}^{+\infty} p b_{n} x^{n+1} y^{n}+\sum_{n=1}^{+\infty} q b_{n} x^{n} y^{n+1}$

Identifying coefficients of $x^{k} y^{l}$ in the above identity, we have the results:

$k=l=0: \quad \alpha u_{00}=0 \Rightarrow u_{00}=0$

Thus $u$ has no constant term.

$k \geq 1, l=0: \quad(k+\alpha) u_{k 0}=0 \Rightarrow u_{k 0}=0$

$k=0, l \geq 1: \quad(-l+\alpha) u_{0 l}=0 \Rightarrow u_{0 l}=0$

$k, l \geq 1,|k-l| \geq 2: \quad(k-l+\alpha) u_{k l}-a(l-1) u_{k-1, l-1}=0 \Rightarrow u_{k l}=0$

This means $u$ does not have any terms like $x^{k} y^{l}$ with $|k-l| \geq 2$. As for the diagonal terms i.e. terms like $x^{k} y^{k}$, we have following results:

$k \geq 2: \quad \alpha u_{k k}-a(k-1) u_{k-1, k-1}=0 \Rightarrow u_{k k}=0$

$k=l=1: \quad \alpha u_{11}=0 \Rightarrow u_{11}=0$

Thus, $u$ does not have any diagonal terms, i.e. terms like $x^{k} y^{k}$. For the rest coefficients we have:

$k=2, l=1: \quad(2-1+\alpha) u_{21}=p b_{1} \Rightarrow u_{21}=\frac{p b_{1}}{1+\alpha}$

$k=1, l=2: \quad(1-2+\alpha) u_{12}-a u_{01}=q b_{1} \Rightarrow u_{12}=\frac{q b_{1}}{-1+\alpha}$

When the integer $k \geq 2$, we have the recursion formulas

$$
\begin{aligned}
& (-1+\alpha) u_{k, k+1}-a k u_{k-1, k}=q b_{k} \Rightarrow u_{k, k+1}=\frac{q b_{k}+a k u_{k-1, k}}{-1+\alpha} \\
& (1+\alpha) u_{k+1, k}-a(k-1) u_{k, k-1}=p b_{k} \Rightarrow u_{k+1, k}=\frac{p b_{k}+a(k-1) u_{k, k-1}}{1+\alpha}
\end{aligned}
$$

Therefore, all coefficients of $u$ can be worked out by coefficients of $f$ and by known constants $a$, $p, q$ and $\alpha$ :

$$
\begin{aligned}
& u_{k, k+1}=\sum_{j=0}^{k-1}\left[\frac{k !}{(k-j) !} \cdot \frac{a^{j} q}{(-1+\alpha)^{j+1}} b_{k-j}\right] \\
& u_{k+1, k}=\sum_{j=1}^{k}\left[\frac{(k-1) !}{(k-j) !} \cdot \frac{a^{j-1} p}{(1+\alpha)^{j}} b_{k-j+1}\right]
\end{aligned}
$$

Here and that follows we regard $0 !=1$. From the preceding induction derivation process, we can see that coefficients of the unknown $u$ are all uniquely determined by given data. So we obtain the conclusion:

The partial differential equation (1) has a unique formal power series solution

$$
u(x, y)=\sum_{k=1}^{+\infty} \sum_{j=0}^{k-1}\left[\frac{(k-1) !}{(k-j-1) !} \cdot \frac{p a^{j} b_{k-j}}{(1+\alpha)^{j+1}} x+\frac{k !}{(k-j) !} \cdot \frac{q a^{j} b_{k-j}}{(-1+\alpha)^{j+1}} y\right](x y)^{k}
$$

Hence, the formal solution $u(x, y)$ could also be written as follows:

$u(x, y)=x u_{1}(x y)+y v_{1}(x y)$

Where $u_{1}(x y)$ and $v_{1}(x y)$ are 


$$
\begin{aligned}
& u_{1}(x y)=\sum_{k=1}^{+\infty} \sum_{j=0}^{k-1}\left[\frac{(k-1) !}{(k-j-1) !} \cdot \frac{p a^{j} b_{k-j}}{(1+\alpha)^{j+1}}\right](x y)^{k} \\
& v_{1}(x y)=\sum_{k=1}^{+\infty} \sum_{j=0}^{k-1}\left[\frac{k !}{(k-j) !} \cdot \frac{q a^{j} b_{k-j}}{(-1+\alpha)^{j+1}}\right](x y)^{k}
\end{aligned}
$$

The uniqueness of the formal solution is obvious since all the coefficients are computed explicitly.

\section{Gevrey order in a monomial}

Let $E$ be a complex Banach space. Let $E \llbracket x \rrbracket$ be the set of all formal power series with coefficients in $E, D_{R}$ be the disc centered at the origin with radius $R>0, \mathcal{O}\left(D_{R} ; E\right)$ be the set of functions which are analytic in $D_{R \text {., }}$ and $E \llbracket t \rrbracket_{s}$ denote the set of $s$-Gevrey series.

Here we cite the definition introduced in [2].

Definition Consider a formal power series $\hat{g}(x, y)=\sum_{m, n=0}^{+\infty} a_{m n} x^{m} y^{n} \in E \llbracket x, y \rrbracket$ and $s>0$. We say that $\hat{g}$ is $s$-Gevrey in the monomial $x y$, if it satisfies the following condition: If $\hat{g}$ is written uniquely as follows

$$
\hat{g}(x, y)=\sum_{n=0}^{+\infty} x^{n} u_{n}(x y)+\sum_{n=1}^{+\infty} y^{n} v_{n}(x y)
$$

With $u_{n}, v_{n} \in E \llbracket t \rrbracket$, then all $u_{n}$ and all $v_{n}$ are s-Gevrey in $t$ and for positive constant $r$ sufficiently small the series $\sum_{n=0}^{+\infty}\left\|\hat{\mathcal{B}}_{s} u_{n}\right\|_{r} x^{n}$ and $\sum_{n=1}^{+\infty}\left\|\hat{\mathcal{B}}_{s} v_{n}\right\|_{r} y^{n}$ converge. Here

$$
\hat{\mathcal{B}}_{s}\left(\sum_{k=0}^{+\infty} a_{k} t^{k}\right)=\sum_{k=0}^{+\infty} a_{k}(k !)^{-s} \tau^{k}
$$

and the norm is defined by $\left\|\sum_{k=0}^{+\infty} a_{k} t^{k}\right\|_{r}=\sum_{k=0}^{+\infty}\left|a_{k}\right| r^{k}$.

Next we'll show that the formal solution in (19) is 1-Gevrey in the monomial $x y$. As it can be written as the form in (20), we only need to prove that both $u_{1}$ and $v_{1}$ are 1-Gevrey in $t(=x y)$ and for constant $r$ sufficiently small the series $\left\|\hat{\mathcal{B}}_{1} u_{1}\right\|_{r} x$ and $\left\|\hat{\mathcal{B}}_{1} v_{1}\right\|_{r} y$ converge.

In fact, let $r$ be a positive constant sufficiently small such that $f(t)$ is bounded at the disc $D_{r}$, i.e. there exists a positive constant $M$ such that each $b_{l}$ in the formula (3) satisfies the inequality

$$
\left|b_{l}\right| \leq \frac{M}{r^{l}}
$$

Then for every positive integer $k$, we may estimate the coefficient of $(x y)^{k}$ as follows

$$
\begin{aligned}
& \left|\sum_{j=0}^{k-1}\left[\frac{(k-1) !}{(k-j-1) !} \cdot \frac{p a^{j} b_{k-j}}{(1+\alpha)^{j+1}}\right]\right| \leq \frac{|p|(k-1) !}{|1+\alpha|} \sum_{j=0}^{k-1} \frac{\left|b_{k-j}\right|}{(k-j-1) !}\left|\frac{a}{1+\alpha}\right|^{j} \\
& \leq \frac{|p| M(k-1) !}{|1+\alpha| r^{k}} \sum_{j=0}^{k-1}\left|\frac{a r}{1+\alpha}\right|^{j} \leq \frac{|p| M}{|1+\alpha|}\left(\frac{1}{r}+\left|\frac{a}{1+\alpha}\right|\right)^{k} k !
\end{aligned}
$$

That is to say $u_{1}$ is 1 -Gevrey in $t(=x y)$. Similarly we could deduce the estimate of coefficients of $v_{1} \quad$ and get the conclusion that $v_{1}$ is 1 -Gevrey in $t(=x y)$.

$$
\begin{aligned}
& \text { Now, } \hat{\mathcal{B}}_{1} u_{1}=\sum_{k=1}^{+\infty} \frac{p}{1+\alpha} \sum_{j=0}^{k-1} \frac{b_{k-j}}{k(k-j-1) !} \cdot\left(\frac{a}{1+\alpha}\right)^{j} \tau^{k} \text {, so } \\
& \left\|\hat{\mathcal{B}}_{1} u_{1}\right\|_{r}=\sum_{k=1}^{+\infty}\left|\frac{p}{1+\alpha} \sum_{j=0}^{k-1} \frac{b_{k-j}}{k(k-j-1) !} \cdot\left(\frac{a}{1+\alpha}\right)^{j}\right| r^{k} \leq \sum_{k=1}^{+\infty} \frac{|p| M}{|1+\alpha| k} \sum_{j=0}^{k-1} \frac{1}{(k-j-1) !} \cdot\left|\frac{a r}{1+\alpha}\right|^{j}
\end{aligned}
$$




$$
\begin{aligned}
& \text { While } \hat{\mathcal{B}}_{1} v_{1}=\sum_{k=1}^{+\infty} \sum_{j=0}^{k-1}\left[\frac{1}{(k-j) !} \cdot \frac{q a^{j} b_{k-j}}{(-1+\alpha)^{j+1}}\right] \tau^{k} \text {, hence } \\
& \left\|\hat{\mathcal{B}}_{1} v_{1}\right\|_{r}=\sum_{k=1}^{+\infty}\left|\frac{q}{-1+\alpha} \sum_{j=0}^{k-1} \frac{b_{k-j}}{(k-j) !} \cdot\left(\frac{a}{-1+\alpha}\right)^{j}\right| r^{k} \leq \sum_{k=1}^{+\infty} \frac{|q| M}{|-1+\alpha|} \sum_{j=0}^{k-1} \frac{1}{(k-j) !} \cdot\left|\frac{a r}{-1+\alpha}\right|^{j}
\end{aligned}
$$

Take $r$ small enough such that the right-hand side series in the above formulas (27) and (28) converge simultaneously. This yields what we want.

\section{Conclusion}

Under our hypotheses, the partial differential equation in two complex variables (1) has a unique formal power series solution which doesn't have any diagonal terms. And this formal solution is 1 -Gevrey in the monomial $x y$.

\section{Acknowledgement}

In this paper, the research was sponsored by the National Natural Science Foundation of China (Project No. 11371071.)

\section{References}

[1] Werner Balser, Masafumi Yoshino, Gevrey order of formal power series solutions of inhomogeneous partial differential equations with constant coefficients, [J]. Funkcial. Ekvac. 2010, 53, 411-434.

[2] Mireille Canalis-Durand, Jorge Mozo-Fernández, Reinhard Schäfke, Monomial summability and doubly singular differential equations, [J]. J. Differential Equations 2007, 233, 485-511.

[3] K. Ichinobe, Summability of formal solution of Cauchy problem for some PDE with variable coefficients, Recent development of micro-local analysis for the theory of asymptotic analysis, [J]. RIMS Kôkyûroku Bessatsu B40 2013, 081-094.

[4] S. Michalik, Analytic solutions of moment partial differential equations with constant coefficients, Funkcial. Ekvac 2013, 56, 19-50.

[5] Alberto Lastra, Stephane Malek, Janier Sanz, On Gevrey solutions of threefold singular nonlinear partial differential equatins, [J]. J. Differential Equations, 2013, 255(10), 3205-3232. 